Revue d'histoire de l'Amérique française

REVUE D.HISTOIRE DE L'AMÉRIQUE FRANÇAISE

\title{
La querelle universitaire québécoise 1876-1889
}

\section{André Lavallée}

Volume 26, numéro 1, juin 1972

URI : https://id.erudit.org/iderudit/303152ar

DOI : https://doi.org/10.7202/303152ar

Aller au sommaire du numéro

Éditeur(s)

Institut d'histoire de l'Amérique française

ISSN

0035-2357 (imprimé)

1492-1383 (numérique)

Découvrir la revue

Citer cet article

Lavallée, A. (1972). La querelle universitaire québécoise 1876-1889. Revue

d'histoire de l'Amérique française, 26(1), 67-81. https://doi.org/10.7202/303152ar d'utilisation que vous pouvez consulter en ligne.

https://apropos.erudit.org/fr/usagers/politique-dutilisation/ 


\title{
LA QUERELLE UNIVERSITAIRE QUÉBÉCOISE $1876-1889^{1}$
}

\author{
ANDré Lavallée \\ Département d'histoire \\ Université du Québec à Montréal
}

Quelques historiens se sont penchés sur la question universitaire québécoise du XIX $\mathrm{X}^{\mathrm{e}}$ siècle: le Père Paul Desjardins, ${ }^{2}$ Robert Rumilly ${ }^{3}$ et Germain Lavallée. ${ }^{4}$ Ils ont sans doute consulté Arthur Savaète qui a reproduit, dans son ouvrage engagé, ${ }^{5}$ une somme de documents aussi considérable que révélatrice. Chez chacun de ces auteurs, nous avons décelé des erreurs d'interprétation communes. Ils ont considéré que cette querelle était d'ordre purement idéologique, entre Ultramontains et Libéraux; ils ne se sont pas suffisamment intéressés à l'étude de la conjoncture; ils ont mal défini l'idéologie libérale en identifiant le libéralisme-conservateur au rougisme. Par conséquent, ils ont tous expliqué cette querelle comme si elle s'était poursuivie sans interruption de 1843 à 1890, sans la replacer dans l'évolution générale du Canada et du Québec et sans accorder l'importance qui convenait aux conjonctures fort différentes de 1843-1870 et 1870-1890, puis au tournant idéologique 1867-1875. Ultramontains VS Libéraux, Montréal VS Québec, Bourget-Laflèche VS Taschereau, telle fut leur thèse commune.

1 Cet article est un résumé de notre thèse de doctorat: Le projet de création d'une université à Montréal (1878-1889): l'opposition entre Montréal et Québec, Ultramontains et Libéraux. Elle a été présentée au département d'Histoire de l'Université de Montréal en 1971.

2 Paul Desjardins, s.j., Le Collège Sainte-Marie de Montréal (Montréal, 1944), 2 vol.; Id. "Question universitaire - Québec-Montréal, contexte historique" (s.l.n.d.). Il s'agit d'un résumé que nous avons consulté, faute de ne pouvoir lire un manuscrit qui devrait constituer un troisième volume de l'ouvrage précédent.

3 Robert Rumilly, "La grande querelle de Laval et de Victoria", L'Hôpital (février à décembre 1937) : 29-32, 153-157, 218-221, 270-275, 314$317,474-478,572-577$.

4 Germain Lavallée, Monseigneur Antoine Racine dans la question universitaire canadienne (1874-1893). Thèse de M. A. (Histoire) présentée à l'Université de Montréal en 1954.

5 Arthur Savaète, Voix canadiennes. Vers l'abîme (Paris, A. Savaète, circa 1908-1918), 12 vol. L'auteur a rédigé cet ouvrage considérable dans un esprit nettement polémiste, prenant la défense des Ultramontains. 
De 1841 à 1875 s'élabora et se noua l'entente entre les autorités civiles et religieuses québécoises. Il devint alors possible pour l'appareil d'Etat de concéder progressivement à l'Eglise qui devint son principal appareil idéologique le monopole de l'éducation qu'elle allait conserver durant plus de trois générations. Après 1840, contrairement aux recommandations d'Arthur Buller inscrites dans le Rapport Durham, ${ }^{6}$ non seulement l'Etat ne mit pas sur pied un système d'éducation "neutre" à vocation essentiellement assimilatrice des Canadiens français, mais il assura la division entre les questions scolaires du Haut et du Bas-Canada, puis il ouvrit la porte à l'éventuel monopole religieux que réalisèrent les législations scolaires, particulièrement celles de 1841, 1846, 1849, 1869 et $1875 .^{7}$ La politique libre-échangiste qu'adopta la métropole anglaise entre 1843 et 1849 amena les modérés des diverses factions politiques du Canada à préparer les modalités d'une superstructure qui se concrétisa avec 1867.8 Ainsi, 1848 avait ouvert la voie à ces forces "d'extrême centre" et rendu possibles, pour ne pas dire inévitables, ${ }^{9} 1867$ et 1931. Au milieu du XIX ${ }^{e}$ siècle, cette élaboration ne se fit pas

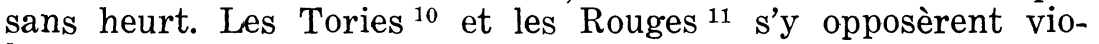
lemment.

C'est en conservant sur les questions d'éducation un schème mental qui s'apparentait fort étrangement à celui de Mgr Hubert et de Mgr Lartigue que les évêques de Montréal et des Trois-

${ }^{6}$ Sir C. P. Lucas, Lord Durham's Report on the Affairs of British North America (Oxford, Clarendon Press, 1912), vol. 3.

7 La loi de 1841 reconnaît le droit de dissidence, celle de 1846 crée deux corporations distinctes, l'une catholique et l'autre protestante, celle de 1849 accorde le droit aux membres du clergé d'être élus commissaires, celle de 1869 accorde en pratique le contrôle de la section catholique du système d'éducation québécois à l'épiscopat et, finalement, celle de 1875 abolit le ministère de l'Instruction publique pour lui substituer la surintendance, consacrant ainsi le caractère clérical et aristocratique du système d'éducation.

8 Stanley B. Ryerson, Unequal Union, Confederation and the Roots of Conflict in the Canadas, 1815-1873 (New York, International Publishers, 1968), 137-191, 244-295, 342-357.

9 Durham, dans son rapport, et Elgin, dans sa volumineuse correspondance, La Collection Elgin-Grey, 1846-1852, éditée avec notes et appendices par Sir Arthur G. Doughty, C. E. B., C.M.G., LL.D. (Ottawa, Imprimeur du Roi, 1937), 4 vol., ont précisément envisagé ces éventualités sans les condamner.

10 Stanley B. Ryerson, ibid., 152-191, 281-295.

11 L'ouvrage récent de Jean-Paul Bernard, Les Rouges. Libéralisme, nationalisme et anticléricalisme au milieu du XIXe siècle (Montréal, $\mathrm{PUQ}$, 1971), envisage bien cette question. 
Rivières, Mgr Bourget et Mgr Laflèche, entrèrent dans cette ère nouvelle du BNA. Dès 1843, Mgr Bourget relança la question universitaire. ${ }^{14}$ En 1851, il précisa ses exigences en matière d'éducation.
A propos de Séminaires et de Collèges, V.G. croirait-elle que le Concile devrait s'occuper de la formation d'une Univer- sité et de l'érection régulière de collèges, dépendant de cette maison-mère d'Education? Ne serait-il pas temps de prendre le devant, et de nous faire constituer légalement maître de l'enseignement catholique ? Pourrait-on refuser au Bas-Canada ce que l'on a accordé au Haut? Le Gouvernement ne serait-il pas forcé par la nécessité des choses, de nous accorder les privilèges ordinaires aux Universités, lorsque l'on ne lui demanderait aucun fonds Provinciaux [sic] pour la dotation de notre Institution qui nous mettrait sur le pied d'égalité avec McGill's University et Queen's College. La crainte que les laïques ne s'emparassent plus tard ici comme en France, de l'éducation ne serait-elle pas une raison puissante pour nous de travailler du moins à réaliser ce projet ? Ne serait-elle pas aussi pour les Séminaires et Collèges un urgent motif de s'entendre à l'amiable entre eux, pour n'être pas exposés au monopole universitaire ? N'est-il pas certain que le Sé- minaire de Québec serait "plaudendibus omnibus", choisi pour être l'Université Catholique de notre Amérique Britan- nique ? Quelle force nous aurions, dans une pareille Institu- tion, après que nous aurions obtenu comme de droit la sanction pontificale? C'est une idée comme une autre que j'envoie à la bonne aventure. Elle fera ou ne fera pas son chemin, c'est autre chose. Je la dépose pour ce qu'elle est aux pieds de Votre Grâce. ${ }^{15}$

Sur un point, et ce n'était certainement pas le moindre en importance, l'évêque de Montréal reprenait un vœu de son prédécesseur, Mgr Lartigue.16 Quant à la crainte des laïques dont il parlait, les gens du Séminaire de Québec la partageaient bien avec lui.

${ }^{14}$ Léon Pouliot, s.j., "L'Enseignement universitaire catholique au Canada français de 1760 à 1860", RHAF, XII (1958) : 155-170.

15 Bourget à Turgeon, le 31 mars 1851, ACAM, RLF, 6 : 398-399.

16 Lartigue à Signay, le 28 mars 1836, ACAM, RLL, 8 : 154-156. Dans cette lettre, on retrouve le passage suivant: "Vous avez grandement raison de dire que l'Evêque de Québec devrait profiter de la chûte [sic] de l'Acte des écoles élémentaires pour recommander partout l'érection des écoles de Fabriques dans chaque Paroisse en vertu des loix [sic] de 1824 et 1827: mais dans une pareille circulaire, il ne doit faire aucune allusion à la chûte [sic] des écoles syndicales, ni témoigner explicitement le désir que nous avons que le clergé s'empare, comme de droit, de l'éducation du peuple." 
Avant que cette question [il s'agissait des conditions d'affiliations des collèges et séminaires à l'Université Laval] soit réglée à la satisfaction de tout le monde, il surgira probablement des petites misères dont il est bon de dérober la connaissance aux laïques, ce que nous ne pourrions plus faire lorsque le Conseil de l'Université serait une fois saisi de cette affaire.

Pour ces raisons, Monseigneur, nous pensons que le plus convenable est de s'entendre d'abord entre prêtres, s'il est possible, et de formuler un plan que les laïques de notre Conseil admettront facilement s'ils sont libres des préjugés qu'aurait fait naître la dissension prématurée et peut-être les exigences de quelques maisons. ${ }^{17}$

Par contre, Mgr Bourget touchait du doigt un problème fondamental qu'il sembla oublier par la suite: le financement de cette entreprise universitaire. ${ }^{18}$ En outre, il minimisa l'importance de la lutte régionale entre Québec et Montréal, lutte qu'il avait pourtant pu constater lui-même dès 1843 .

Quant à la réflexion qui termine votre lettre du 16 courant, savoir que je veux trainer (sic) votre Diocèse à la remorque, je vous sais bon gré et vous remercie de bon cœur de cet avis, que vous voulez bien me donner. Je dois pourtant entrer en quelque explication avec V.G. à ce sujet. Si je comprends bien la signification de cette expression: trainer (sic) à la remorque, j'aurais la prétention de vouloir vous forcer à faire dans votre Diocèse tout ce que je fais ici. Assurément, Monseigneur, cette folie ne m'est jamais venue en tête. Si pressé par les besoins de cette partie de la vigne du Seigneur confiée à mes soins, j'ai cru devoir entreprendre quelque chose, je n'ai jamais pensé que vous dûssiez en faire autant. Je me suis alors contenté de vous informer, parce que en certaines occasions vous m'aviez paru mortifié de ce que je ne vous avais pas prévenu de quelques innovations opérées ici. Je vous dirai franchement que lorsqu'au (sic) meilleur de ma conscience, je crois devoir entreprendre quelque chose pour l'avantage de ce Diocèse, qui ne s'accorde pas avec vos vues, je regarderais comme une politique tortueuse que de faire semblant de vous consulter, lorsque je serais décidé à agir contrairement à votre opinion. Je désire beaucoup m'entendre avec vous pour toutes les mesures uniformes, qui peuvent

17 Casault à Bourget, le 29 avril 1854, ACAM, Université, 820.001,

18 Mémoire sur l'Université Laval avec pièces justificatives (Québec, 1862) ; Mémoire présenté par le Séminaire de Québec à NN. SS. les Evêques de la province de Québec, assemblés aux Trois-Rivières, en octobre 1864 (s.l.n.d.). En outre, l'ouvrage du Père Paul Desjardins que nous avons mentionné plus haut, de même que celui de Savaète, qui vont au-delà de 1864, nous fournissent de nombreux exemples à ce sujet. 
tendre au bien général des deux Diocèses. Ainsi je me suis employé et je m'emploie encore de mon mieux pour que le St Siège érige ici une Province Ecclésiastique, afin que je devienne votre Suffragant. (...) Mais faut-il, pour ne pas mériter d'être accusé de vouloir trainer (sic) votre Diocèse à la remorque, que je m'assujétisse à ne rien faire d'important sans votre avis et sans votre approbation, c'est ce que je ne pense pas; . . .19

Peut-être plus idéaliste encore et plus épris des combats épiques, Mgr Laflèche continua la lutte contre les "égarés" après la démission de Mgr Bourget. ${ }^{20}$ L'aile libérale-conservatrice du clergé québécois travailla autant qu'elle avait dû le faire pour Mgr Bourget afin d'obtenir la démission de Mgr Laflèche. ${ }^{21}$ Contrairement à Mgr Bourget, et sans renverser ciel et terre lorsque la chose se produisit, l'évêque des Trois-Rivières allait un jour accepter ce qu'il avait pourtant considéré comme devant être le plus grand échec de sa vie et le moins justifiable: la division de son diocèse. ${ }^{22}$

Les enquêteurs pontificaux, Mgr Conroy ${ }^{23}$ et Mgr Smeulders, ${ }^{24}$ ne comprirent qu'en partie la conjoncture et les intérêts

19 Bourget à Signay, le 27 mars 1843, ACAM, RLB, $3: 13-16$.

20 Bourget à Franchi, le 8 avril 1876, ACAM, RLB, $25: 53-55$.

21 Dans une lettre à T.-E. Hamel, Mgr Antoine Racine lui suggère qu'on fasse une pétition pour démitrer Mgr Laflèche. Voir A. Racine à Hamel, le 10 novembre 1881, dans Germain Lavallée, Monseigneur Antoine Racine, 29. T.-E. Hamel ne tarda pas à lui répondre : "Enfin, si V. G. écrit au Cardinal Siméoni, ne pourrait-elle pas insister sur l'impossibilité de gouverner la Province de Québec tant que Mgr Laflèche conservera sa mitre administrative ? [...] Un petit coup d'épaule de votre part qui ressemblerait à un motu proprio arraché par les événements, appuierait beaucoup l'Archevêque." Voir Hamel à Racine, le 15 novembre 1881, dans ibid., 29.

22 En 1885, Rome nomma Mgr Cameron d'Antigonish dont la mission consista principalement à effectuer la division du diocèse des Trois-Rivières. C'est alors que fut érigé le diocèse de Nicolet dont le premier évêque fut Mgr Gravel. Voir Robert Rumilly, Monseigneur Laflèche et son temps (Montréal, s.l.n.d.), 269-292. Mgr Dominique Racine tenait à cette division parce que c'était "le plus sûr moyen d'obtenir sa démission" d'autant plus qu'il avait "paraît-il, déclaré à diverses reprises que si son diocèse était divisé, il résignerait de suite". Voir D. Racine à Taschereau, le 8 novembre 1881, dans Jean-Claude Drolet, Monseigneur Dominique Racine, bâtisseur de l'Eglise saguenéenne (Publications de la Société historique du Saguenay, 1968), 135.

23 Afin de hâter l'exécution du décret du $1^{\text {er }}$ février 1876, Rome délégua au Canada Mgr George Conroy d'Irlande. Ce dernier arriva au Canada au mois de mai 1877 , mais il n'aborda qu'à la fin de l'été l'étude de la question universitaire. Voir Paul Desjardins, "Question universitaire".

24 Abbé mitré de l'Ordre des Cisterciens, consulteur de la Propagande, le Commissaire Smeulders arriva à Québec le 21 octobre 1883. Son enquête 
en cause dans la querelle universitaire. Le premier comprit que le libéralisme à l'européenne dont Mgr Bourget, Mgr Laflèche et les Jésuites accusaient leurs adversaires et particulièrement Mgr Taschereau et les gens du Séminaire de Québec n'existait pas au Québec. Par contre, il ne sembla pas comprendre le fondement économique de l'opposition entre Québec et Montréal. Pour sa part, Dom Henri Smeulders saisit bien l'opposition régionale, il soupçonna l'existence d'un problème financier, mais il l'interpréta mal; il croyait qu'avec ses richesses immobilières le Séminaire de Québec pouvait et devait assurer la viabilité de l'Université Laval à Québec sans avoir besoin de compter sur la succursale montréalaise. Enfin, Mgr Smeulders se convainquit trop facilement de ce que la nature de l'opposition régionale était purement idéologique et doctrinale.

Face à la politique lavalloise, les Jésuites et les Sulpiciens, ayant des statuts civils différents, ne pouvaient pas réagir de façon identique. En mesure d'aider financièrement l'implantation de l'Université Laval à Montréal et étant incorporés civilement, les Sulpiciens purent faire reconnaître leur statut particulier. Ils négocièrent peu de temps ${ }^{25}$ et obtinrent dès le printemps 1878,26 pour la faculté de Théologie montréalaise, des conditions pratiquement identiques à celles que les Jésuites ne devaient obtenir pour leurs collèges qu'en $1889 .{ }^{27}$ En retour, ils offrirent à

fut arrêtée d'une façon tragique. Mgr Taschereau se rendit à Rome au mois de mai 1884 et n'en revint que le $1^{\text {er }}$ décembre. Il avait réussi à obtenir le désaveu de Dom Smeulders qui dut quitter le Canada sans remettre de rapport final au Saint-Siège. Nous avons traité en détail cette question compliquée dans notre thèse, pages 188-254.

25 Les négociations entre les Sulpiciens et l'Université Laval concernant la faculté de Théologie ne durèrent que du mois de novembre 1877 au mois d'avril 1878. Les Sulpiciens obtinrent pratiquement tout ce qu'ils demandèrent dans un document préparé à l'automne. Voir "Mémoire sur les conditions posées par le Séminaire de Montréal pour l'érection d'un faculté de Théologie de l'Université Laval au Grand Séminaire de Montréal", autographe de M. Colin, p.s.s., fin d'octobre ou début de novembre [sic], ASS, Université, C. 17, no 8 .

26 "Entente entre le Séminaire de Québec et l'Université Laval d'une part, et le Séminaire de Saint-Sulpice de Montréal, d'autre part, sur les conditions de la création de la faculté de Théologie du Grand Séminaire de Montréal", document original signé par J.-A. Baille, sup., Thos.-E. Hamel, p.s.s.q., J.-B. Larue, p.s.s., L. Colin, p.s.s., A.-J.-P. Légaré, ptre, M.-P. Méthot, ptre, le 2 avril 1878, ASS, Université, C. 17, no 15.

27 "Mémoire de M. de Troie, p.s.s., supérieur du séminaire, sur les circonstances de la création de la faculté de Théologie de l'université Laval à Montréal, en 1878", ASS, C. 17, no 10, document autographe de M. Troie, p.s.s., 1878, non signé. Dans ce document, on retrouve le passage suivant : "Le Supérieur de Montréal nommait les professeurs, les auteurs ... 
la faculté de Droit de la succursale leur cabinet de lecture "éclairé et chauffé", plus un don de $\$ 1000 .{ }^{28}$ ce qui permettait à cette section lavalloise de s'installer à Montréal. Sûrs d'euxmêmes, avant que ne fussent achevées leurs négociations sur cette question, les Sulpiciens demandèrent la permission d'établir, avec les mêmes conditions, des facultés de Philosophie et de Droit canon. ${ }^{29}$ Comme on leur refusa cette concession, ils n'acceptèrent pas d'affilier leur Collège de Montréal à Laval avant $1886,{ }^{30}$ c'est-à-dire avant d'être assurés d'avoir la direction du Collège canadien à Rome, ${ }^{31}$ ce qui allait faire d'eux les responsables de la formation de l'élite cléricale canadienne.

La situation financière, l'ultramontanisme et le statut juridique des Jésuites compliquèrent considérablement leur cas. Il fut impossible de le résoudre en dehors d'un règlement d'ensemble embrassant à la fois la question universitaire, les grands débats "idéologiques" que connaissait la province de Québec surtout depuis la décennie 1840 et les difficultés majeures que rencontra le Canada au cours des années 1870 et 1880 . En ce qui concerne la question des biens des Jésuites et celle de l'Université, déjà, en 1876, le sort de la Compagnie de Jésus pouvait être prévisible en bonne partie. La politique anglicisatrice de leurs supérieurs de New York avait monté contre eux les dirigeants laïques, l'épiscopat et une bonne partie de la population francophone. ${ }^{32}$

Tout dépendait de Montréal, des degrés aussi, seulement les Diplomes [sic] officiels seraient demandés à Québec, qui n'aurait rien à faire dans l'administration de Montréal."

28 Thos.-E. Hamel, "Notes pour servir à l'histoire du Séminaire de Québec et de l'Université Laval", ASQ, Manuscrit 33, p. 11 (le 5 avril 1878). 29 "Mémoire de M. L. Colin, directeur du séminaire [sic], sur le projet d'ériger canoniquement une faculté de Théologie à Montréal, qui serait affiliée à l'Université Laval", le 2 janvier 1878, ASS, Université, C. 17, $\mathrm{n}^{0} 11$.

30 "Brevet d'affiliation du Collège de Montréal à l'Université Laval de Québec" [original en latin], le 20 janvier 1887, ASS, Collège de Montréal, T. $48, \mathrm{n}^{\circ} 171$.

31 "Rapport d'un comité du Conseil législatif approuvé par le lieutenant-gouverneur le 12 juin 1886, accordant l'autorisation demandée", les 9 et 12 juin 1886, ASS, Collège canadien à Rome, C. 21, nº 38.

32 De 1842 à 1869 , la mission jésuite du Québec était rattachée à la province française de Champagne. En 1869, une mission indépendante fut constituée en Amérique. Elle portait le nom de mission New YorkCanada. Jusqu'en 1879, les Jésuites du Québec en firent partie. En 1876, les Jésuites du Québec se plaignirent de la politique suivie par leurs provinciaux américains. Voir Vignon à Charaux, le 23 décembre 1876, dans Léon Pouliot, s.j. "Lents et pénibles débuts de la Province du Canada français: le problème linguistique", Lettres du Bas-Canada, XXII (mars 1969) : 78-93. 
En plus d'avoir aggravé sérieusement leur situation financière, ${ }^{33}$ la construction du Collège Sainte-Marie et celle du Gesu avait fait s'élever les Sulpiciens contre eux. ${ }^{34}$ L'ultramontanisme du Père Braun ${ }^{35}$ et le rapport que ne manquèrent pas d'établir Mgr Langevin, Mgr Taschereau et Mgr LaRoque entre la demande d'une charte universitaire ${ }^{36}$ et les démarches du Père

33 En 1871 , le Collège Sainte-Marie accusait un déficit de $\$ 200,000$. Voir "La Compagnie de Jésus au Canada: Mémoire adressé au T.R.P. Général”, ACSiM, Sér. MC, Portf. 8 : n 16, p. 20.

34 Les Sulpiciens songèrent à ériger un externat à l'angle des rues Sherbrooke et Bleury en guise de représailles contre la construction du Collège Sainte-Marie. L'intervention de Mgr Bourget les dissuada de mettre leur plan à exécution. Voir Bourget à Charbonnel, le 6 novembre 1845, ACAM, RLB, 4:59; Charbonnel à Bourget, 7-10 novembre 1845, ACAM, Jésuites; $i d$. à $i d$., le 10 novembre 1845, ACAM, Jésuites; $i d$. ., à $i d$. ., le 12 novembre 1845, ACAM, Jésuites; "La Compagnie de Jésus au Canada", 19.

35 En 1872, année des noces d'or de Mgr Bourget, des fêtes furent organisées en deux temps : une manifestation de caractère intime dans la chapelle de l'évêché pour le personnel de l'évêché et les plus chers amis de Mgr Bourget, et une grande démonstration publique à Notre-Dame. A la chapelle, on avait d'abord choisi comme predicateur le Pere sraun, un Jésuite reconnu pour son attachement à la doctrine du Syllabus; mais il fut remplacé par l'abbé Alexis Pelletier. Ce dernier, après s'être brouillé avec Mgr Taschereau, avait été admis dans le diocèse de Montréal où il continua à iutter pour la cause ultramontaine. A Notre-Dame, c'était Mgr Laflèche qui devait prononcer le sermon. Comme il se trouva absent au dernier moment, on demanda au Père Braun de le remplacer. Pris au dépourvu, le Jésuite n'eut pas le temps de préparer un autre texte que celui dont il aurait dû se servir s'il avait fait le sermon à la chapelle de l'évêché. Mais, selon son habitude, il avait déjà mémorisé son texte. Ainsi, à Notre-Dame, il prononça le sermon qu'il avait préparé en tenant compte de ceux qui étaient présents à la chapelle de l'évêché et non pas de tous les personnages qui pouvaient se trouver à Notre-Dame. Il loua donc le zèle de Mgr Bourget à répandre la doctrine du Syllabus et attaqua tous ceux qui se considéraient comme libéraux au sens où on entendait ce terme à Laval et à l'archevêché de Québec, "libéral-conservateur". Mgr Taschereau, qu'on n'attendait pas à la chapelle de l'évêché, arriva juste à temps pour entendre le sermon de l'abbé Pelletier, et, à Notre-Dame, il écouta le sermon du Père Braun. En quelques jours, les "libéraux" et Mgr Taschereau en particulier se sentirent attaqués et critiqués violemment à Montréal. Jamais ils ne pardonnèrent au Père Braun et à ses supérieurs montréalais qui l'appuyaient ce sermon des noces d'or. Voir: Paul Desjardins, Le Collège Sainte-Marie de Montréal, 2 : 286.

36 Pendant que l'Ecole de Droit de l'Institut canadien fondée par Joseph Doutre, en 1866, et affiliée à McGill en 1871, affermissait ses positions et se vantait de posséder autant d'étudiants que les autres universités, une nouvelle faculté de Médecine, affiliée au Bishop's College de Lennoxville, ouvrait ses portes à Montréal. En 1872, de jeunes professionnels, médecins et avocats, signèrent deux requêtes à l'intention de Mgr Bourget pour le prier de s'occuper de la fondation d'une université catholique indépendante à Montréal. A ce moment-là, la question des écoles du Nouveau-Brunswick était à l'étude en Chambre. Elle provoquait des 
Charaux relatives à un règlement éventuel de la question des biens ${ }^{37}$ expliquèrent la conduite du recteur de Laval en $1878 .{ }^{38}$ Les Lavallois ne voulaient pas d'un règlement de la question des biens qui aurait été de nature à renflouer facilement les coffres des Jésuites et qui aurait peut-être fait d'eux des adversaires trop puissants parce que trop riches et indépendants de l'archevêque de Québec. Ils souhaitaient une entente qui aurait permis au Séminaire de Québec de combler le déficit causé par l'expérience universitaire et qui aurait garanti la bonne marche

déclarations contradictoires sur les devoirs des députés catholiques. $\mathrm{Mgr}$ Bourget crut le moment venu d'intervenir à Rome pour demander son université. Ses idées étaient bien arrêtées. Il les fit connaître à Mgr Desautels, curé de Varennes. "Une des conséquences [de l'attitude des évêques de Québec et de Rimouski dans la question des écoles du Nouveau-Brunswick], c'est que l'Université Laval va se trouver entachée de gallicanisme. Vous en profiterez sans doute pour montrer la nécessité d'établir à Montréal une université qui sera assez ultramontaine pour tenir en bride celle de Laval. [...] Vous pourrez attester que nous pouvons ... organiser et mettre sur un bon pied l'Université en projet, qui sera connue sous le nom d'université-Pie, et qui aura pour principale mission de combattre et d'extirper de notre heureux pays toutes les erreurs condamnées par cet immortel et intrépide Pontife. Mais il faut traiter de cette affaire avec tout le secret possible, pour éviter tous les embarras que ne manquerait pas de nous susciter l'Université Laval." Dans cet état d'esprit, Mgr Bourget demanda au Collège Sainte-Marie de se prêter, comme corps civilement constitué, à des démarches en vue d'obtenir de la législature le pouvoir de conférer les grades académiques en Droit et en Médecine. Mais les démarches de Mgr Taschereau auprès des autorités romaines eurent pour conséquence que la Propagande ordonna aux Jésuites de cesser de solliciter une charte universitaire auprès du gouvernement de Québec. Voir Paul Desjardins, Le Collège Sainte-Marie de Montréal, 2 : 270-300.

37 Voir "La Compagnie de Jésus au Canada", 22.

38 Contrairement à la recommandation inscrite dans le décret pontifical du $1^{\text {er }}$ février 1876 à l'effet que tout l'épiscopat de la province devait collaborer à l'implantation de la succursale Laval à Montréal, le recteur Hamel se contenta de se servir de Mgr Fabre comme intermédiaire entre l'Université et les Jésuites. Il leur présenta un Modus vivendi qu'ils ne pouvaient accepter sans abandonner du même coup leur Ratio Studiorum. Ce document, "le Modus vivendi", accompagné d'une lettre explicative, est reproduit in extenso dans "Lavallensis Succursalis", 148. Quelques années plus tard, en présence de plusieurs témoins, le recteur de l'Université aurait déclaré à celui de Sainte-Marie : "Nous ne voulions pas des PP. Jésuites dans la succursale de l'Université à Montréal; mais Mgr Conroy a pensé qu'il valait mieux vous donner la faculté des Arts." Voir "Lettre du Père Cazeau", le 4 octobre 1882, ACSM, Sér. MC, Port. 6, $n^{\circ}$ 9. Et le Père Cazeau jura que "Mgr Fabre m'a dit que Mons. Louis Pâquet lui avait dit: s'ils [les messieurs de Laval] eussent cru que les Jésuites devraient consentir à faire partie de la Succursale, ils auraient posé d'autres conditions." Voir Louis Pâquet à Fabre, s.d., dans Vignon, "Lavallensis Succursalis", 151. 
de cette entreprise pour les siècles des siècles. ${ }^{39}$ Aussi, refusèrentils de reconnaître, comme ils venaient de le faire pour les Sulpiciens, un statut particulier pour les Jésuites. Ils repoussèrent les conditions "sine qua non" mises de l'avant par ces religieux ${ }^{40}$ pour s'occuper de la faculté des Arts de la succursale. Les Lavallois ne demandèrent même pas aux Jésuites, comme l'avait recommandé Mgr Conroy, ${ }^{41}$ de mettre sur pied la faculté de Droit à Montréal. Jusqu'à la fin des années 1880, les Jésuites ne se trouvèrent pas incorporés civilement, ce qui les plaça dans une position plus que difficile pour tenter de négocier directement avec les autorités civiles un règlement de la question des biens. Les Lavallois, et particulièrement Mgr Taschereau, ${ }^{42}$ allaient s'efforcer de profiter au maximum de cette faiblesse. Il n'y eut pas de faculté des Arts jésuite à Montréal, ${ }^{43}$ ni règlement de la question des biens, ni incorporation de la Compagnie de Jésus, tant et aussi longtemps que la double conjoncture canadienne et québécoise ne le permit, c'est-à-dire: pas avant la deuxième moitié de la décennie 1880 .

39 Taschereau à Légaré, 19-22 août 1884, ASQ, Séminaire : 9, no 4q.

40 Fabre à Hamel, le 15 janvier 1878, ACAM, RLF, $3: 54^{\mathrm{e}}$ feuillet. Par l'intermédiaire de Mgr Fabre, les Jésuites demandèrent à Laval : “... un modus vivendi clair, précis, qui règle nos rapports tant avec les évêques qu'avec l'Université-Mère, une sorte de contrat qui, tout en établissant une certaine subordination aussi nécessaire que juste, ne lèse en rien la dignité ou la liberté de notre Compagnie et de ses supérieurs..."

41 Vignon, "Lavallensis Succursalis", 142.

42 En 1887, Mgr Taschereau fit tout ce qu'il put afin que la question des biens soit réglée avant que les Jésuites ne soient incorporés civilement. Voir Roy C. Dalton, The Jesuits' Estates Question, 1760-1888 (University of Toronto Press, 1968), 145-152.

43 Autant que les Jésuites, les Sulpiciens refusaient d'affilier à Laval leur Collège de Montréal parce qu'ils ne voulaient pas être soumis aux règlements du Conseil universitaire concernant les programmes et les examens. Cependant, lorsqu'ils furent assurés qu'on leur confierait le Collège canadien à Rome et qu'ils se mirent à craindre une perte de prestige par rapport aux Jésuites, dans la province de Québec, ils conclurent plusieurs ententes successives avec Laval. En échange de la présidence de la faculté des Arts, Louis Colin, p.s.s., fit accepter à ses supérieurs la nécessité de l'affiliation du Collège de Montréal. Voir Mémoire sur l'affiliation du Collège de Montréal à l'Université Laval intitulé : "Sommesnous obligés de nous affilier à l'Université Laval ?", vers 1880 [sic], ASS, Collège de Montréal, T. 48, no 137; Méthot à Colin, le 10 septembre 1886, ASS, Université, C. $17, n^{\circ} 37$; Colin à Icard, le 26 octobre 1886, dans ibid., $n^{\circ} 38$; Colin à Méthot et Marcoux, 3-5 janvier 1887, dans ibid., $\mathrm{n}^{\circ} 39$; "Entente entre le Séminaire de Québec et le Séminaire de Saint-Sulpice de Montréal concernant la faculté des Arts de l'Université Laval à Montréal et l'affiliation du Collège de Montréal à cette université" [confidentiel], le 4 janvier 1887, dans ibid., n ${ }^{\circ} 40$. 
C'est à tort, croyons-nous, que le Père Paul Desjardins et Robert Rumilly ont considéré les dirigeants de l'Ecole de Médecine et de Chirurgie de Montréal de même que les religieuses de l'Hôtel-Dieu comme des Ultramontains convaincus. Depuis plusieurs années, ${ }^{44}$ quelques médecins de l'Ecole s'étaient obligés financièrement envers les religieuses de l'Hôtel-Dieu qui leur avaient prêté les sommes nécessaires à l'achat d'un terrain et à la construction de la bâtisse où logea l'Ecole. Ni les religieuses ni ces médecins-professeurs ne tenaient à perdre cet argent dont le montant atteignait les $\$ 25,000.45$ Tous ces intéressés refusèrent donc d'obéir aux décrets pontificaux de 1876, 1881 et 1883 qui ordonnaient à l'Ecole de se fondre dans la faculté de Médecine de la succursale sans tenir compte de ce fait capital. A l'instar des Sulpiciens, pour ce qui est de l'affiliation de leur Collège de Montréal, et des Jésuites en ce qui concerne la question de leurs biens, de leur incorporation civile et de l'affiliation du Collège Sainte-Marie, l'Ecole de Médecine et l'Hôtel-Dieu durent attendre une conjoncture favorable pour voir se régler leurs problèmes.

La position de Mgr Fabre fut sans doute l'une des plus inconfortables dans cette affaire universitaire. Et les Lavallois refusèrent péremptoirement d'en tenir compte.46 Succéder à Mgr Bourget et refuser de lui être inféodé, ce n'était pas chose facile. Le nouvel évêque en était conscient et ne se fit pas d'illusion. ${ }^{47} \mathrm{Mgr}$ Bourget avait constitué un chapitre dont la grande majorité des membres lui étaient aveuglément dévoués; il avait fait de la querelle universitaire l'une de ses principales préoccupations; il abandonna son siège alors que le diocèse se trouvait pratiquement en faillite, ${ }^{48}$ puis il continua d'intervenir

44 Le 4 mars 1884, la supérieure de l'Hôtel-Dieu signalait quelques dates, 1860,1872 et 1882 , relatives à des contrats signés avec l'Ecole, mais elle précisait qu'elle en ignorait quelques-unes sous prétexte qu'elles n'avaient pas été consignées dans les livres des délibérations du conseil de l'hôpital. Voir Savaète, Voix canadiennes, 5 : 224. En vertu d'un indult pontifical, Mgr Bourget avait permis et approuvé ces contrats. Cet indult portait sur l'aliénation des biens ecclésiastiques et avait été signé par le cardinal Siméoni, Préfet de la Propagande, le 27 mars 1870. Voir ce document dans ibid., 226, note 1 .

45 Savaète, ibid., $4: 225$, note 1.

46 "Journal de Mgr Michel-E. Méthot", 12 décembre 1881, ASQ, Manuscrit 16, 51 et 53.

47 E.-J. Auclair, Saint-Henri des tanneries de Montréal (Montréal, 1942), 47.

48 En 1879 , la dette de l'évêché atteignait plus de $\$ 800,000$. et les revenus du diocèse étaient insuffisants pour en payer seulement les intérêts. 
dans la lutte ${ }^{49}$ jusqu'à sa mort qui survint en 1885. Dans une conjoncture économique difficile, Mgr Fabre dut affronter son chapitre et une bonne partie de son clergé qui l'embarrassèrent autant dans ses efforts en vue de restaurer la situation financière de son diocèse que dans ses démarches relatives à la question universitaire.

Ainsi Mgr Méthot, de l'Université Laval, écrivait à ThomasEtienne Hamel :

Je suis allé voir Mgr (i.e. Fabre) qui m'a fait grand pitié. Il passe actuellement par une grande crise. Plusieurs chanoines veulent partir, et il a exigé leur démission de chanoine. Il ne peut pas les laisser partir immédiatement, à cause de son départ et des embarras financiers de l'Evêché. Il ne sait où trouver des hommes de confiance pour les remplacer. La situation financière de l'Evêché est telle que, si elle était connue, la banqueroute s'ensuivrait. Il se plaint beaucoup de ce que, parmi tout cela, on veuille l'obliger de payer l'affaire de la Faculté de Médecine et de l'Hôtel-Dieu, ce qui va exaspérer encore les chanoines et le clergé ! L'Archevêque l'aurait menacé de monter à Montréal régler lui-même la question. ${ }^{50}$

Sous les feux de la rampe, il ne pouvait satisfaire à la fois aux exigences des décrets pontificaux, de l'archevêque de Québec et des Lavallois, puis contenter tous ses diocésains : les banquiers, ses chanoines, ses curés, les médecins de l'Ecole et les religieuses de l'Hôtel-Dieu. Acceptant sa soumission à Rome autant que Mgr Taschereau, il fut conséquent au point de se mériter la censure pontificale en 1883 pour un excès de zèle. ${ }^{.1}$ Pourtant, dans l'ensemble, il sembla très réaliste. Dès son premier voyage ad limina en 1879-1880, il avait exposé au Saint-Siège ce qui était en quelque sorte l'unique façon de trancher le débat universitaire : accorder à Montréal une autonomie la plus complète qui soit par rapport à Québec en élevant son diocèse au rang d’archevêché et dégager alors la succursale montréalaise

Voir le mémoire de J.-B. Champeau, S. Tassé, J. Primeau, N. Piché, A.-P. Dubuc et O.-J. Devlin, "A Sa Sainteté Léon XIII", Montréal, 26 octobre 1882, aux archives de l'Archevêché de Montréal.

$49 \mathrm{Au}$ printemps 1879 , Mgr Bourget écrivit à Mgr Fabre une lettre qui commençait par ces mots : "Je n'ai pas oublié que sans moi vous ne seriez pas Evêque de Montréal..." Bourget à Fabre, le 19 mai 1879, ACAM, Chapitre-Cathédrale, 319.300.

50 Méthot à Hamel, le 18 mai 1878, Séminaire 59, nº $37 \mathrm{~b}$.

51 Télégramme de Siméoni à Fabre, le 25 août 1883, ACAM, Université, Médecine, 831.106. 
d'une soumission complète à Laval tout en assurant le contrôle religieux. ${ }^{52}$

Une solution d'ensemble ne devait s'avérer réalisable que dans une conjoncture favorable, celle des années 1885-1890. La pendaison de Riel, les remous qu'elle souleva au Québec et au Canada anglais, ${ }^{53}$ de même qu'un commencement de relance économique, provoquèrent un réalignement des forces en présence qui permit enfin le règlement des grandes querelles québécoises dont celle de l'Université. La crise économique qui avait précipité les événements du Nord-Ouest ${ }^{54}$ avait aussi rendu difficile l'assainissement des finances du diocèse de Montréal. L'affaire Riel avait relancé de plus belle la querelle entre Canadiens français et Canadiens anglais. ${ }^{55}$ Pour calmer l'agitation québécoise au lendemain de l'exécution politiquement intéressée du chef métis, ${ }^{56}$ qui risquait de compliquer tragiquement la situation des francophones des autres provinces et particulièrement ceux de l'Ontario, politiciens et ecclésiastiques misèrent sur le sentiment religieux des Canadiens français.

D’Ottawa Mgr Duhamel écrivait à Mgr Taschereau et à Mgr Fabre:

Des hommes haut placés et qui connaissent le sentiment public de la province d'Ontario, surtout des environs de Toronto, des catholiques qui s'effrayent des maux qui en résulteraient pour les Canadiens, craignent des contre-démonstrations et prévoient le danger d'une guerre de race, m'ont demandé hier soir s'il n'y aurait pas moyen, pour les Evêques, de ramener le calme. J'ai promis d'en écrire à Votre Grandeur qui, en sa qualité de Métropolitain, peut donner une direction ou appeler à une réunion les Evêques de la Province.

Les catholiques des autres provinces du Dominion ne semblent pas prêts à s'unir à nos Canadiens sur la question qui est discutée un peu partout dans la province de Québec et beaucoup dans les grands centres.

$\mathrm{Si}$ les Evêques se tiennent complètement à l'écart de cette circonstance, je crains qu'ils perdent beaucoup de leur in-

52 Siméoni à Fabre, le 25 février 1880, ACAM, RLF, 1 : 172-176; Pâquet à Hamel, le 6 février 1880, ASQ, Université $73, \mathrm{n}^{\circ} 76$.

$53 \mathrm{On}$ peut lire à ce sujet G.F.G. Stanley, The Birth of Western Canada: A History of the Riel Rebellion (Toronto, 1963), 380-407.

54 Ibid., 177-294.

55 Nous avons précisément étudié cette question dans notre Mémoire de D.E.S. en Histoire à l'Université de Montréal : La Rébellion de 1885 dans le Nord-Owest canadien: les réactions canadiennes et "canadian". 56 G. F. G. Stanley, The Birth of Western Canada, 79-99. 
fluence et qu'à l'avenir il leur devienne impossible d'arrêter les mouvements populaires, alors même qu'ils seraient dirigés contre l'autorité légitime. ${ }^{57}$

Ils utilisèrent toutes leurs influences à Ottawa, à Londres, à Westminster et à Rome ${ }^{58}$ et obtinrent du Saint-Siège l'élévation de l'archevêque de Québec au rang de cardinal. ${ }^{59}$ Afin que cet honneur n'aggravât pas davantage l'antipathie des Montréalais contre les Québécois, Rome érigea Montréal en archevêché. ${ }^{60}$ Mgr Fabre accepta d'autant plus facilement cet arrangement, attendu d'ailleurs depuis longtemps, que le problème financier de son diocèse était presque résolu. ${ }^{61}$ L'indépendance s'acquérait ainsi Montréal par rapport à Québec et l'intelligence qu'avaient alors les autorités romaines des possibilités financières du gouvernement provincial rendirent possibles le règlement de la question des biens des Jésuites ${ }^{62}$ et la bulle Jamdudum ${ }^{63}$ peu de temps après que la Compagnie de Jésus eut obtenu de Québec une loi d'incorporation civile. ${ }^{64}$

Ainsi, entre 1876 et 1890 , la rivalité traditionnelle entre Québec et Montréal et les difficultés financières constituèrent bel et bien la trame de fond de la querelle universitaire. L'opposition entre Libéraux et Ultramontains était plus qu'anachronique; c'était, à toute fin pratique, un faux problème. Mgr Taschereau, Mgr Fabre, les gens du Séminaire de Québec, les Lavallois et la plupart de leurs alliés n'étaient pas plus libéraux que Mgr Bourget, Mgr Laflèche, les religieuses de l'Hôtel-Dieu et les professeurs de l'Ecole de Médecine et de Chirurgie. Ils

57 Duhamel à Fabre, le 27 novembre 1885, ACAM, Ottawa, 255.110.

58 Charles Langelier, Souvenirs politiques (Québec, 1909), 325-326; Ouimet à Macdonald, le 27 novembre 1885, APC, Sir John A. Macdonald Papers; Casgrain à Macdonald, le 24 décembre 1885, dans ibid.; Macdonald à Taschereau, le 10 mars 1886, dans ibid.; le supérieur du Collège français à Rome à Mgr Taschereau, le 10 mars 1886, ACAM, RLF, 6 : 168-169.

59 Voir D. C. Lyne, "Sir John A. Macdonald and the Appointment of Canada's First Cardinal", Revue d'études canadiennes, II, 4 (novembre 1967) : 58-60.

60 "Bref de Sa Sainteté Léon XIII érigeant Montréal en Archevêché et nommant Mgr E.-C. Fabre archevêque de Montréal", le 8 juin 1886, Documents relatifs à l'érection de Montréal en archevêché, RCD, 91 ; "Circulaire au clergé d'Ottawa", le 22 juin 1886, dans ibid.

61 Le 19 mars 1887, Mgr Fabre allait rédiger une circulaire pour expliquer que les finances de son diocèse étaient revenues à la normale. Voir MDM, 10 : 328-330.

62 Roy C. Dalton, The Jesuits' Estates Question, 163.

63 MDM, 10 : 546-554.

64 Dalton, ibid., 152. 
s'étaient adaptés à la conjoncture, à la réalité nouvelle des années 1840 et 1850 et particulièrement à celles des années qui suivirent 1867 ; ils étaient en somme de bons libéraux-conservateurs. Les "dangers" qui avaient justifié l'action des Ultramontains et de Mgr Bourget au lendemain de 1840, l'offensive des Rouges et le prosélytisme anglo-protestant n'existaient pratiquement plus. Les Rouges avaient été balayés par le système confédératif et par la condamnation romaine de 1869. Les lois scolaires, principalement celles de 1869 et 1875, avaient fait du clergé, ce dont avaient rêvé $\mathrm{Mgr}$ Lartigue et Mgr Bourget, le maître incontesté du système d'éducation de la province de Québec. Il ne restait plus dès lors, en réalité, que des Lavallois et des Montréalistes accablés par des difficultés financières, difficultés que seule la conjoncture favorable des années $1885-1890$ pouvait résoudre de façon normale et satisfaisante. 